\title{
CANONICABILITY OF THE FIRST ORDER SYSTEM OF HYPERBOLIC EQUATIONS
}

\author{
MANSUR I. ISMAILOV AND IBRAHIM TEKIN \\ Communicated by Fioralba Cakoni
}

\begin{abstract}
The paper establishes a condition for the first order hyperbolic system (with a boundary condition at infinity) to have a solution that can be expressed by the second kind Volterra operator. On the basis of this condition, some canonical forms (for instance Dirac-type system) of the first order hyperbolic system can be sorted. The suitability of this condition in inverse scattering problem for the first order hyperbolic system on the half line is given as an application.
\end{abstract}

1. Introduction. The Volterra type integral representation of the solution plays an important role in solving inverse scattering problems (ISP's). Such a representation can be taken from a transformation operator (TO) at infinity.

Transformation operators were introduced in the fifties by Gelfand, Levitan and Marchenko in connection with the ISP for the SturmLiouville (S-L) operator $[\mathbf{1 4}, \mathbf{1 6}]$. In the classical case, TO maps the solution of nonperturbed S-L equation into the solution of perturbed S-L equation (the so-called Jost solution). In this case, the TO has the form of the identity operator plus a certain Volterra integral operator. Such an integral representation of the solution was the basis of the investigation of the ISP for the system of ordinary differential equations $[7,13]$.

The TO method is also suitable for inverse problems for partial differential equations. This method is successfully applied to ISP for

2010 AMS Mathematics subject classification. Primary 45D05, Secondary 35L50, 35P25.

Keywords and phrases. Inverse scattering problem, Scattering data, First order hyperbolic system.

Received by the editors on November 4, 2013, and in revised form on May 8, 2014.

DOI: $10.1216 /$ JIE-2015-27-1-47

Copyright (일 2015 Rocky Mountain Mathematics Consortium 
the first order hyperbolic system of two equations (two component nonstationary Dirac equation) by Nizhnik [18]. In contrast to this case, the first order system of $n(n>2)$ equations with the boundary conditions at $x \rightarrow+\infty$ has a complicated structure (the TO is matrix integral operator but not of Volterra type) [19]. This makes it difficult to solve the ISP for such systems. Some particular cases where the system of hyperbolic equations has the Volterra type integral TO are considered in $[9,11]$.

Consider the following first order system of hyperbolic equations on the half line $x \geq 0$ :

$$
\sigma \frac{\partial \psi}{\partial t}-\frac{\partial \psi}{\partial x}=Q(x, t) \psi, \quad t \in \mathbb{R},
$$

where $\sigma=\operatorname{diag}\left\{\xi_{1}, \ldots, \xi_{n}\right\}$ is a diagonal matrix of constants with

$$
\xi_{1} \geq \cdots \geq \xi_{m}>0>\xi_{m+1} \geq \cdots \geq \xi_{n},
$$

and $Q(x, t)$ is matrix function with measurable complex-valued rapidly decreasing (Schwartz) entries.

Our goal in this paper is to determine the conditions for which system (1) has Volterra type TO at $x \rightarrow+\infty$ and its possible application to ISP for the first order system of hyperbolic equations on the half line. We will call system (1) which has TO at $x \rightarrow+\infty$ in the form of the identity operator plus certain Volterra integral operator as canonicable.

The ISP for system (1) on the whole line was studied extensively in [18] by utilizing a Gelfan-Levitan-Marchenko (GLM) type linear integral equation and in [23] by utilizing a Riemann-Hilbert problem. Generally, the ISP's on the half line are ill-posed because they have fundamental difficulties in determining the sufficient quantity of scattering problems (on the half line for the same system of equations) for ensuring the unique solution of the ISP under consideration. Some of the particular cases are studied in $[\mathbf{9}, \mathbf{1 1}]$ but the mathematical formulation of the ISP's for the system (1) on the half line in general is an open problem. The canonicability of the system (1) is important because its scattering operator on the half line has Volterra type factorization (Krein's factorization) which plays a significant role in solving of the ISP on the half line. By using such type of factorizations, the ISP is reduced to the matrix integral equations which are known as the GLM type linear integral equations. Detailed information about Krein's the- 
ory on factorization of the Fredholm operators of the second kind can be found in $[8,12,18]$.

The system of first order hyperbolic equations (1) describes the multi-velocity interaction (with the velocities $\xi_{k}, k=\overline{1, n}$ ) of the waves in a nonstationary media. Hence, the coefficients of the relevant partial differential equation vary not only in space, but also in time. Model problems, where time-dependent coefficients occur, can be found in electromagnetic wave theory, acoustics and in optics, $[\mathbf{1}, \mathbf{2 4}]$. The traditional underlying model for wave propagation, in general, is the wave equation with coefficients independent of time. As examples, we can mention a model of interacting cable lines in electric engineering and the Timoshenko model of a beam in elasticity theory. The relevant system of these models is a two velocity second-order hyperbolic system with only spatially varying coefficients. The inverse problem of finding the coefficients of such a type of systems from response operator (or input-output correspondence) is investigated in $[4, \mathbf{5}, \mathbf{1 7}, \mathbf{2 0}]$. It is important to notice that the paper [4] observes some effects (presence of a precursor, shaping the waves, etc.) of bringing up interaction between waves. This paper also considers the ISP on the axis with the symmetric potential localized on a finite interval $0<x<h$. It is proved that potential can be recovered via the scattering data which includes reflection and transition coefficients of the fundamental matrix with $x \leq 0$ and $x \geq h$, respectively. The present paper and the papers $[\mathbf{9}, \mathbf{1 1}, \mathbf{1 2}, \mathbf{1 8}]$ also investigate the ISP for the multi-velocity system (in particular, for the one- and two-velocity systems) for the more general case because the potential is time-dependent and is not localized on a finite interval. However, the statement and scattering data of the ISP studied in these papers differ significantly from those used in [4] where the ISP is solved by constructing the response operator via scattering data.

Notice also that, if the potential is independent of $t$, then by taking $\psi(x, t)=\psi(x) \exp (i \lambda t)$ with the separation of variables, we can convert (1) into the first order system of ordinary differential equations given by $(d / d x) \psi(x)+Q(x) \psi(x)=i \lambda \sigma \psi(x)$. The inverse problem for this system is discussed satisfactorily in $[2,3,6,7,13,15,21,25]$.

The ISP for system (1) is also of great interest as auxiliary problem for some integrable multidimensional nonlinear evolution equations. Some of nonlinear evolution equations related to the first order strictly 
hyperbolic systems are given in $[\mathbf{1 8}, \mathbf{2 3}]$. One can also notice that, in the paper [10], the ISP for a first order non-strictly hyperbolic system is applied to integration of the continual $N$-wave interaction problem.

The paper is organized as follows.

In Section 2, the conditions of having the Volterra type TO (the canonicability) for the first order hyperbolic system with the boundary condition at $x \rightarrow+\infty$ are established. In Section 3, some examples (canonical forms) are given. In Section 4, the ISP on the half plane for the canonical system of first order hyperbolic equations is considered and the preliminary Volterra property of the scattering operator is obtained. The value of this property in inverse scattering problem for the canonicable first order hyperbolic system on the half plane is given as an example. Finally, in the appendix, the solvability of a Volterra type integral equation is given.

2. Transformation operators for the first order hyperbolic system with the boundary conditions at infinity. Consider system (1), with $\psi(x, t)=\operatorname{col}\left\{\varphi_{1}(x, t), \ldots, \varphi_{n}(x, t)\right\}$, and $Q(x, t)=$ $\left(q_{k j}\right)_{k, j=1}^{n}$. In the case of $Q(x, t)=0$ in system (1), it is easy to verify that this system has the solution

$$
\psi_{0}(x, t)=\Im_{\sigma x} h(t)
$$

where $h(t)$ is an arbitrary vector function,

$$
\Im_{\sigma x}=\operatorname{diag}\left\{T_{\xi_{1}}, \ldots, T_{\xi_{n}}\right\}
$$

is a shift operator such that

$$
T_{\xi_{i}} h(t)=h\left(t+\xi_{i} x\right), \quad i=1, \ldots, n .
$$

Let us consider system (1) under the boundary condition

$$
\lim _{x \rightarrow+\infty}\left(\psi(x, t)-\psi_{0}(x, t)\right)=0 .
$$

We shall denote the space of $\mathbb{C}^{n}$ valued bounded continuous functions on $\mathbb{R}$ as $C_{b}\left(\mathbb{R}, \mathbb{C}^{n}\right)$.

It is similar to [23, Proposition 3.1] that, for an arbitrary vector $h \in C_{b}\left(\mathbb{R}, \mathbb{C}^{n}\right)$, there exists a unique, bounded and continuous solution of the problem (1)-(3). Really, problem (1)-(3) is equivalent to the 
following system of integral equations:

$$
\psi(x, t)=\Im_{\sigma x} h(t)+\int_{x}^{+\infty} \Im_{\sigma(x-\eta)}[Q \psi](\eta, t) d \eta .
$$

This system of integral equations can be reduced into the form

$$
\begin{aligned}
\varphi_{k}(x, t)= & h_{k}\left(t+\xi_{k} x\right) \\
& +\int_{-\infty}^{0} \sum_{j=1}^{n} q_{k j}\left(x-\tau, t+\xi_{k} \tau\right) \varphi_{j}\left(x-\tau, t+\xi_{k} \tau\right) d \tau, \\
& k=1, \ldots, n .
\end{aligned}
$$

This system obviously satisfies condition Lemma 6.1 (i) (see appendix) with $(\alpha, \beta)=(-1,0)$ and $\left(\alpha_{j}, \beta_{j}\right)=\left(-1, \xi_{k}\right)$. This system also satisfies Lemma 6.1 (ii) because the entries $q_{k j}$ of the matrix $Q(x, t)$ belong to the Schwartz class. Therefore, Lemma 6.1 is applicable for yielding unique solvability.

Now, let us suppose that the problem (1)-(3) (i.e., system of integral equations (4)) has a solution in the form

$$
\psi(x, t)=\psi_{0}(x, t)+\int_{x}^{+\infty} A(x, t, s) \psi_{0}(s, t) d s .
$$

Denote

$$
\begin{aligned}
& \sigma_{=}=\left\{(k, j): \xi_{k}=\xi_{j}\right\} ; \\
& \sigma_{+}=\left\{(k, j): \frac{\xi_{j}-\xi_{k}}{\xi_{j}}>0\right\} ; \\
& \sigma_{-}=\left\{(k, j): \frac{\xi_{j}-\xi_{k}}{\xi_{j}}<0\right\} .
\end{aligned}
$$

If the solution of (4) can be represented in the form (5) for each $h \in C_{b}\left(\mathbb{R}, \mathbb{C}^{n}\right)$, then substituting (5) into (4), we obtain the system of equations for the kernels:

$$
\begin{gathered}
A_{k j}(x, t, s)=\sum_{p=1}^{n} \int_{x}^{+\infty} q_{k p}\left(\eta, t+\xi_{k}(x-\eta)\right) \\
A_{p j}\left(\eta, t+\xi_{k}(x-\eta), s-x+\eta\right) d \eta, \\
(k, j) \in \sigma_{=}, \quad s \geq x
\end{gathered}
$$


(6) $\quad A_{k j}(x, t, s)=\frac{\xi_{j}}{\xi_{j}-\xi_{k}} q_{k j}\left(\frac{\xi_{j} s-\xi_{k} x}{\xi_{j}-\xi_{k}}, t+\xi_{k}\left(x-\frac{\xi_{j} s-\xi_{k} x}{\xi_{j}-\xi_{k}}\right)\right)$

$$
\begin{gathered}
+\sum_{p=1}^{n} \int_{x}^{\left(\xi_{j} s-\xi_{k} x\right) /\left(\xi_{j}-\xi_{k}\right)} q_{k p}\left(\eta, t+\xi_{k}(x-\eta)\right) \\
A_{p j}\left(\eta, t+\xi_{k}(x-\eta), s-\frac{\xi_{k}}{\xi_{j}}(x-\eta)\right) d \eta \\
(k, j) \in \sigma_{+}, \quad s \geq x \\
A_{k j}(x, t, s)=\sum_{p=1}^{n} \int_{x}^{+\infty} q_{k p}\left(\eta, t+\xi_{k}(x-\eta)\right) \\
A_{p j}\left(\eta, t+\xi_{k}(x-\eta), s-\frac{\xi_{k}}{\xi_{j}}(x-\eta)\right) d \eta, \\
(k, j) \in \sigma_{-}, \quad s \geq x
\end{gathered}
$$

and the following relations between kernels $A_{k j}$ and coefficients $q_{k j}$, $(k, j) \in \sigma_{=}$or $(k, j) \in \sigma_{-}$of the system $(1)$ :

$$
\begin{gathered}
q_{k j}(x, t)=0, \quad(k, j) \in \sigma_{=} \\
q_{k j}(x, t)=\frac{\xi_{j}-\xi_{k}}{\xi_{j}} \sum_{p=1}^{n} \int_{x}^{+\infty} q_{k p}\left(\eta, t+\xi_{k}(x-\eta)\right) \\
A_{p j}\left(\eta, t+\xi_{k}(x-\eta), x-\frac{\xi_{k}}{\xi_{j}}(x-\eta)\right) d \eta, \\
s \leq x, \quad(k, j) \in \sigma_{-} .
\end{gathered}
$$

It is easy to show that the system of equations (6) can be reduced into the following forms:

$$
\begin{gathered}
A_{k j}(x, t, s)=\int_{-\infty}^{0} \sum_{p=1}^{n} q_{k p}\left(x-\tau, t+\xi_{k} \tau\right) \\
A_{p j}\left(x-\tau, t+\xi_{k} \tau, s-\tau\right) d \tau, \\
(k, j) \in \sigma_{=},
\end{gathered}
$$




$$
\begin{gathered}
A_{k j}(x, t, s)=\frac{\xi_{j}}{\xi_{j}-\xi_{k}} q_{k j}\left(\frac{\xi_{j} s-\xi_{k} x}{\xi_{j}-\xi_{k}}, t+\xi_{k}\left(x-\frac{\xi_{j} s-\xi_{k} x}{\xi_{j}-\xi_{k}}\right)\right) \\
+\int_{-\xi_{j}(s-x) /\left(\xi_{j}-\xi_{k}\right)}^{0} \sum_{p=1}^{n} q_{k p}\left(x-\tau, t+\xi_{k} \tau\right) \\
A_{p j}\left(x-\tau, t+\xi_{k} \tau, s-\frac{\xi_{k}}{\xi_{j}} \tau\right) d \tau \\
(k, j) \in \sigma_{+}, \\
A_{k j}(x, t, s)=\int_{-\infty}^{0} \sum_{p=1}^{n} q_{k p}\left(x-\tau, t+\xi_{k} \tau\right) \\
A_{p j}\left(x-\tau, t+\xi_{k} \tau, s-\frac{\xi_{k}}{\xi_{j}} \tau\right) d \tau \\
(k, j) \in \sigma_{-} .
\end{gathered}
$$

By using Lemma 6.1 with $(\alpha, \beta)=(-1,0)$ and $\left(\alpha_{p}, \beta_{p}\right)=\left(-1, \xi_{k}\right)$, this system has a unique bounded solution.

In this situation, equalities (7) are the conditions for which system (1) has a Volterra solution in the form of (5).

Therefore, the following theorem is proved:

Theorem 2.1. System (1) under condition (3) has the bounded solution in the form of (5) if and only if the kernels of the integral operator in (5) satisfy (6) and the coefficients and kernels have relations (7).

3. Some canonical forms. In this section, we will establish two examples for which system (1) has Volterra solution in the form of (5). We will obtain two canonical forms of system (1) in the case where the relations (7) are satisfied automatically.

Rewrite system (1) in its suitable form:

$$
\begin{aligned}
\sigma_{1} \partial_{t} \psi_{1}-\partial_{x} \psi_{1} & =Q_{11}(x, t) \psi_{1}+Q_{12}(x, t) \psi_{2}, \\
\sigma_{2} \partial_{t} \psi_{2}-\partial_{x} \psi_{2} & =Q_{21}(x, t) \psi_{1}+Q_{22}(x, t) \psi_{2}
\end{aligned}
$$

with $\sigma_{1}=\operatorname{diag}\left\{\xi_{1}, \ldots, \xi_{m}\right\}, \sigma_{2}=\operatorname{diag}\left\{\xi_{m+1}, \ldots, \xi_{n}\right\}$. Here, $\psi_{1}$ stands for the first $m$ components, $\psi_{2}$ stands for the last $n-m$ components, 
respectively, of the $n$ component solution $\psi=\operatorname{col}\left\{\psi_{1}, \psi_{2}\right\}$ of (1), and

$$
Q=\left[\begin{array}{ll}
Q_{11} & Q_{12} \\
Q_{21} & Q_{22}
\end{array}\right]
$$

where $Q_{11}$ is $m \times m, Q_{12}$ is $m \times(n-m), Q_{21}$ is $(n-m) \times m$ and $Q_{22}$ is $(n-m) \times(n-m)$ matrices which have measurable complex-valued entries belonging to the Schwartz class.

Example 3.1 (Dirac-type system [12]). Let $\xi_{1}=\cdots=\xi_{m}=1$, $\xi_{m+1}=\cdots=\xi_{n}=-1$. If $Q_{11}=Q_{22}=0$, then system (8) is called the Dirac-type system [21]. Although this type of system is called Zakharov-Shabat (ZS) ([6, 15]), Ablowitz-Kaup-Newell-Segur (AKNS) ([2]), Hamiltonian ([3]) or canonical ([22]) systems in various literature. In this case, system (8) has a solution in the form

$$
\psi(x, t)=\psi_{0}(x, t)+\int_{x}^{+\infty}\left[\begin{array}{ll}
A_{11} & A_{12} \\
A_{21} & A_{22}
\end{array}\right](x, t, s) \psi_{0}(s, t) d s
$$

where $A_{11}, A_{12}, A_{21}$ and $A_{22}$ are $m \times m, m \times(n-m),(n-m) \times m$, $(n-m) \times(n-m)$ matrices, respectively, and satisfy the following system of integral equations (see [12])

$$
\begin{aligned}
A_{11}(x, t, \tau)= & \int_{x}^{+\infty} Q_{12}(s, x+t-s) A_{21}(s, x+t-s, \tau-x+s) d s, \\
A_{21}(x, t, \tau)= & \frac{1}{2} Q_{21}\left(\frac{\tau+x}{2}, t+\frac{\tau-x}{2}\right) \\
& -\int_{(\tau+x) / 2}^{x} Q_{21}(s, t-x+s) A_{11}(s, t-x+s, \tau+x-s) d s, \\
\tau \geq & x, \quad Q_{(\tau+x) / 2} Q_{12}(s, t+x-s) A_{22}(s, t+x-s, \tau+x-s) d s, \\
A_{12}(x, t, \tau)= & \frac{1}{2} Q_{12}\left(\frac{\tau+x}{2}, t+\frac{\tau-x}{2}\right) \\
& -\int_{x}^{x} Q_{21}(s, t-x+s) A_{12}(s, t-x+s, \tau-x+s) d s, \\
\tau \geq & x,
\end{aligned}
$$

and the relations (7) are satisfied automatically. 
Example 3.2 (TS structure system [11]). Let $n=2 m$ and $\xi_{1}>\cdots>$ $\xi_{m}>0>\xi_{m+1}>\cdots>\xi_{2 m}$. Suppose that the matrix $Q$ is given by the following triangular structure:

$Q_{11}$ is a strictly lower triangular $m \times m$ matrix function, $Q_{12}$ is a lower anti-triangular $m \times m$ matrix function, $Q_{21}$ is an upper antitriangular $m \times m$ matrix function and $Q_{22}$ is a strictly upper triangular $m \times m$ matrix function.

In this case, system (8) has a solution in the form

$$
\psi(x, t)=\psi_{0}(x, t)+\int_{x}^{+\infty}\left[\begin{array}{ll}
A_{11} & A_{12} \\
A_{21} & A_{22}
\end{array}\right](x, t, s) \psi_{0}(s, t) d s,
$$

where $A_{11}$ is lower triangular, $A_{12}$ is lower anti-triangular, $A_{21}$ is upper anti-triangular and $A_{22}$ is an upper triangular $m \times m$ matrix kernel. Denote

$$
\begin{aligned}
& A_{11}(x, t, s)=\sigma_{1} B_{11}\left(x, t, t+\sigma_{1}(s-x)\right), \\
& A_{12}(x, t, s)=-\sigma_{2} B_{12}\left(x, t, t+\sigma_{2}(s-x)\right), \\
& A_{21}(x, t, s)=\sigma_{1} B_{21}\left(x, t, t+\sigma_{1}(s-x)\right), \\
& A_{22}(x, t, s)=-\sigma_{2} B_{22}\left(x, t, t+\sigma_{2}(s-x)\right),
\end{aligned}
$$

where the constant matrix $\sigma=\operatorname{diag}\left\{\xi_{1}, \ldots, \xi_{n}\right\}$ is applied to the $k$ th $(k=\overline{1, n})$ column of $\sigma A(x, t, t+\sigma(s-x))$ as $\xi_{k} A_{k i}\left(x, t, t+\xi_{k}(s-x)\right)$, $i=\overline{1, n}$. The kernels $B_{i j}(x, t, \tau), i, j=1,2$, satisfy the following system of integral equations (see [11])

$$
\begin{aligned}
{\left[B_{11}\right]_{i j}(x, t, \tau)=} & \frac{1}{\xi_{j}-\xi_{i}}\left[Q_{11}\right]_{i j}\left(x+\frac{\tau-t}{\xi_{j}-\xi_{i}}, t-\frac{\xi_{i}}{\xi_{j}-\xi_{i}}(\tau-t)\right) \\
& +\int_{x}^{x+(\tau-t) /\left(\xi_{j}-\xi_{i}\right)}\left[Q_{11} B_{11}+Q_{12} B_{12}\right]_{i j} \\
& \left(s, t+\xi_{i}(x-s), \tau+\xi_{j}(x-s)\right) d s \\
{\left[B_{21}\right]_{i j}(x, t, \tau)=} & \frac{1}{\xi_{j}-\xi_{m+i}}\left[Q_{21}\right]_{i j} \\
& \left(x+\frac{\tau-t}{\xi_{j}-\xi_{m+i}}, t-\frac{\xi_{m+i}}{\xi_{j}-\xi_{m n+i}}(\tau-t)\right) \\
& +\int_{x}^{x+(\tau-t) /\left(\xi_{j}-\xi_{m+i}\right)}\left[Q_{21} B_{11}+Q_{22} B_{21}\right]_{i j}
\end{aligned}
$$




$$
\begin{aligned}
& \left(s, t+\xi_{m+i}(x-s), \tau+\xi_{j}(x-s)\right) d s, \quad \tau \geq x \\
{\left[B_{12}\right]_{i j}(x, t, \tau)=- } & \frac{1}{\xi_{m+j}-\xi_{i}}\left[Q_{12}\right]_{i j} \\
& \left(x+\frac{\tau-t}{\xi_{m+j}-\xi_{i}}, t-\frac{\xi_{i}}{\xi_{m+j}-\xi_{i}}(\tau-t)\right) \\
+ & \int_{x}^{x+(\tau-t) /\left(\xi_{m+j}-\xi_{i}\right)}\left[Q_{12} B_{22}+Q_{11} B_{12}\right]_{i j} \\
{\left[B_{22}\right]_{i j}(x, t, \tau)=- } & \frac{\left(s, t+\xi_{i}(x-s), \tau+\xi_{m+j}(x-s)\right) d s}{\xi_{n+j}-\xi_{n+i}}\left[Q_{22}\right]_{i j} \\
& \left(x+\frac{\tau-t}{\xi_{m+j}-\xi_{m+i}}, t-\frac{\xi_{m+i}}{\xi_{m+j}-\xi_{m+i}}(\tau-t)\right) \\
+ & \int_{x}^{x+(\tau-t) /\left(\xi_{m+j}-\xi_{m+i}\right)}\left[Q_{21} B_{12}+Q_{22} B_{22}\right]_{i j} \\
& \left(s, t+\xi_{m+i}(x-s), \tau+\xi_{m+j}(x-s)\right) d s, \quad x \geq \tau,
\end{aligned}
$$

where $[B]_{i j}(x, t, \tau)$ denotes the $i, j$ element of the matrix function $B(x, t, \tau)$ and $[Q B]_{i j}(s, t, \tau)=[Q(s, t) B(s, t, \tau)]_{i j}$, and the relations (7) are satisfied automatically. When $i=j$, the upper bound of the integral is $+\infty$.

The matrix $\left(a_{i j}\right)_{i, j=1}^{n}$ is called lower (strictly) triangular, if $a_{i j}=0$ for all $j>i$ (for all $j \geq i$ ); lower anti-triangular, if $a_{i j}=0$ for all $j+i \leq n$; upper (strictly) triangular, if $a_{i j}=0$ for all $j<i$ (for all $j \leq i)$; and upper anti-triangular, if $a_{i j}=0$ for all $j+i \geq n+2$.

4. A Volterra structure of the scattering operator on the half-plane for the first order hyperbolic system. Consider system (8) under the following boundary condition on $x=0$ :

$$
\psi_{2}(0, t)=H \psi_{1}(0, t)
$$

where $H$ is the $(n-m) \times m$ constant matrix and following asymptotic relation

$$
\psi_{1}(x, t)=\Im_{\sigma_{1} x} a(t)+o(1), \quad x \rightarrow+\infty,
$$

where $a(t)$ defines the profile of the incident waves. 
The problem (8)-(10) is called the scattering problem on the halfplane. The following theorem holds for the scattering problem:

Theorem 4.1. For an arbitrary vector $a(t) \in C_{b}\left(\mathbb{R}, \mathbb{C}^{m}\right)$, there exists a unique solution of the scattering problem, and the second component of the solution satisfies the asymptotic relation

$$
\psi_{2}(x, t)=\Im_{\sigma_{2} x} b(t)+o(1), \quad x \rightarrow+\infty,
$$

where $b(t) \in C_{b}\left(\mathbb{R}, \mathbb{C}^{n-m}\right)$ defines the profile of the scattered waves.

Proof. The scattering problem (8)-(10) is equivalent to the following system of integral equations:

$$
\begin{aligned}
& \psi_{1}(x, t)=\Im_{\sigma_{1} x} a(t)+\int_{x}^{+\infty} \Im_{\sigma_{1}(x-s)}\left[Q_{11} \psi_{1}+Q_{12} \psi_{2}\right](s, t) d s, \\
& \psi_{2}(x, t)=\Im_{\sigma_{2} x} b(t)+\int_{x}^{+\infty} \Im_{\sigma_{2}(x-s)}\left[Q_{21} \psi_{1}+Q_{22} \psi_{2}\right](s, t) d s,
\end{aligned}
$$

where

$$
\begin{aligned}
& b(t)=H a(t) \\
& +\int_{0}^{+\infty}\left\{H \Im_{-\sigma_{1} s}\left[Q_{11} \psi_{1}+Q_{12} \psi_{2}\right](s, t)-\Im_{-\sigma_{2} s}\left[Q_{21} \psi_{1}+Q_{22} \psi_{2}\right](s, t)\right\} d s .
\end{aligned}
$$

From this, it follows that

$$
\begin{aligned}
\varphi_{i}(x, t)= & a_{i}\left(t+\xi_{i} x\right) \\
& +\frac{1}{\xi_{i}} \int_{-\infty}^{t} \sum_{j=1}^{n}\left[q_{i j} \varphi_{j}\right]\left(x+\frac{t-\tau}{\xi_{i}}, \tau\right) d \tau, \quad i=1, \ldots, m, \\
\varphi_{m+l}(x, t)= & \sum_{i=1}^{m} h_{l i} a_{i}\left(t+\xi_{m+l} x\right) \\
& +\int_{-\infty}^{t} r_{m+l}(x, t, \tau) d \tau, \quad l=1, \ldots, n-m,
\end{aligned}
$$


where

$$
r_{m+l}(x, t, \tau)=\left\{\begin{array}{c}
\sum_{i=1}^{m}\left(h_{l i}\right) /\left(\xi_{i}\right) \sum_{j=1}^{n}\left[q_{i j} \varphi_{j}\right]\left(\frac{\xi_{m+l}}{\xi_{i}} x+\frac{t-\tau}{\xi_{i}}, \tau\right), \\
\tau<t+\xi_{m+l} x, \\
\frac{1}{\xi_{m+l}} \sum_{j=1}^{n}\left[q_{m+l, j} \varphi_{j}\right]\left(x+\frac{t-\tau}{\xi_{m+l}}, \tau\right), \\
\tau>t+\xi_{m+l} x
\end{array}\right.
$$

Since the above system of integral equations is Volterrian in $t$ and kernels belong to the Schwartz class, the solution of this system exists and is unique (see [11]).

The relation (11) follows from (12) by virtue of the coefficients $q_{i j}(x, t), i, j=1, \ldots, n$ belonging to the Schwartz class.

According to Theorem 4.1, for every incident wave vector $a(t) \in$ $C_{b}\left(\mathbb{R}, \mathbb{C}^{m}\right)$, there is a bounded solution of the scattering problem, and this solution defines the scattering wave vector $b(t) \in C_{b}\left(\mathbb{R}, \mathbb{C}^{n-m}\right)$ by (11). Thus, we can define in space $C_{b}\left(\mathbb{R}, \mathbb{C}^{n-m} \times \mathbb{C}^{m}\right)$ the matrix operator $S_{H}$ translating $a$ to $b$ :

$$
b=S_{H} a .
$$

This operator is called the scattering operator on the half-plane for system (8) with boundary condition (9).

The canonicability of system (8) makes the structure of the operator $S_{H}$ a Volterra structure.

Theorem 4.2. Let $S_{H}$ be the scattering operator of system (8) which is canonicable (satisfies condition (7)). Then $S_{H}$ has given the factorization

$$
S_{H}=\left(I+A_{+}\right)\left(H+A_{-}\right),
$$

where $I$ is identity operator, $A_{-}$is the upper Volterra integral operator and $A_{+}$is lower Volterra integral operator.

Proof. We take into account that $h(t)=\operatorname{col}\{a(t), b(t)\}$. By using a change of variables, rewrite representation (5) as follows:

$$
\psi_{1}(x, t)=\Im_{\sigma_{1} x} a(t)+\int_{t}^{+\infty} \widehat{A}_{11}(x, t, s) \Im_{\sigma_{1} x} a(s) d s
$$




$$
\begin{aligned}
& +\int_{-\infty}^{t} \widehat{A}_{12}(x, t, s) \Im_{\sigma_{2} x} b(s) d s \\
\psi_{2}(x, t)= & \Im_{\sigma_{2} x} b(t)+\int_{t}^{+\infty} \widehat{A}_{21}(x, t, s) \Im_{\sigma_{1} x} a(s) d s \\
& +\int_{-\infty}^{t} \widehat{A}_{22}(x, t, s) \Im_{\sigma_{2} x} b(s) d s
\end{aligned}
$$

where $\widehat{A}_{11}(x, t, s)$ is $m \times m, \widehat{A}_{12}(x, t, s)$ is $m \times(n-m), \widehat{A}_{21}(x, t, s)$ is $(n-m) \times m$ and $\widehat{A}_{22}(x, t, s)$ are $(n-m) \times(n-m)$ matrix kernels which are denoted as follows:

$$
\begin{aligned}
& \widehat{A}_{11}(x, t, s)=\frac{1}{\sigma_{1}} A_{11}\left(x, t, x+\frac{1}{\sigma_{1}}(s-t)\right), \\
& \widehat{A}_{12}(x, t, s)=-\frac{1}{\sigma_{2}} A_{12}\left(x, t, x+\frac{1}{\sigma_{2}}(s-t)\right), \\
& \widehat{A}_{21}(x, t, s)=\frac{1}{\sigma_{1}} A_{21}\left(x, t, x+\frac{1}{\sigma_{1}}(s-t)\right), \\
& \widehat{A}_{22}(x, t, s)=-\frac{1}{\sigma_{2}} A_{22}\left(x, t, x+\frac{1}{\sigma_{2}}(s-t)\right) .
\end{aligned}
$$

Here the constant matrix $\sigma=\operatorname{diag}\left\{\xi_{1}, \ldots, \xi_{m_{1}}\right\}$ is applied to the $k$ th $\left(k=\overline{1, m_{1}}\right)$ column of $m_{2} \times m_{1}$ matrix $(1 / \sigma) A(x, t, x+(1 / \sigma)(s-t))$ as $\left(1 / \xi_{k}\right) A_{k i}\left(x, t, x+\left(1 / \xi_{k}\right)(s-t)\right), i=\overline{1, m_{2}}$.

From the boundary condition and representation (15), we understand that

$$
b(t)+A_{21-} a(t)+A_{22+} b(t)=H a(t)+H A_{11-} a(t)+H A_{12+} b(t),
$$

or

$$
\left(I+A_{22+}-H A_{12+}\right) b(t)=\left(H+H A_{11-}-A_{21-}\right) a(t),
$$

where

$$
\begin{gathered}
A_{i 1-}(x) f(t)=\int_{t}^{+\infty} \hat{A}_{i 1}(x, t, s) f(s) d s, \\
A_{i 2+}(x) f(t)=\int_{-\infty}^{t} \hat{A}_{i 2}(x, t, s) f(s) d s, \\
A_{i 1-}=A_{i 1-}(0), \quad A_{i 2+}=A_{i 2+}(0), \quad i=1,2 .
\end{gathered}
$$


By comparing the definition of scattering operator (13) and the equalities (15), we get

$$
S_{H}=\left(I+A_{+}\right)\left(H+A_{-}\right),
$$

where $I+A_{+}=\left(I+A_{22+}-H A_{12+}\right)^{-1}$ and $A_{-}=H A_{11-}-A_{21-}$.

The Volterra structure (14) of the scattering operator $S_{H}$ is important as a solution of ISP for the first order hyperbolic system on the half plane.

For example, in the case $n=2 m$, consider two scattering problems on the semi-axis for the canonicable system (8) with boundary conditions:

$$
\psi_{2}(0, t)=H_{k} \psi_{1}(0, t), \quad \operatorname{det} H_{k} \neq 0, \quad k=1,2 .
$$

It is known (see $[\mathbf{1 1}, \mathbf{1 9}])$ that the transmission matrix operator

$$
\Pi=\left[\begin{array}{ll}
A_{11-} & A_{12+} \\
A_{21-} & A_{22+}
\end{array}\right]
$$

is a scattering operator on the whole-axis for a system of first order hyperbolic equations, with the coefficients of system (8) equal to zero for $x<0$. Since the inverse scattering problem for the system of hyperbolic equations on the whole-axis is solved in $[\mathbf{1 8}, \mathbf{2 3}]$, then we obtain the following result about the ISP for the canonicable system (8) on the semi-axis in the case $n=2 m$.

Theorem 4.3. Let $S_{H_{1}}, S_{H_{2}}$ be two scattering operators on the semiaxis for the canonicable system (8) with $n=2 m$, where the matrices $H_{1}, H_{2}$ satisfy condition $\operatorname{det}\left[H_{1}-H_{2}\right] \neq 0$. Then the coefficients of system (8) are uniquely determined by the scattering operators $S_{H_{1}}$ and $S_{H_{2}}$.

Proof. Denoting $B_{k+}=A_{22+}-H_{k} A_{12+}$ and $B_{k-}=H_{k} A_{11-} H_{k}^{-1}-$ $A_{21-} H_{k}^{-1}$ and using the equality $S_{H_{k}} H_{k}^{-1}=I+F_{k}(k=1,2)$, we obtain from (14) that

$$
B_{k-}=B_{k+}+F^{k}+B_{k+} F^{k} .
$$

The kernels of the integral operators $B_{k-}, B_{k+}$ involved in (16) are denoted by $B_{k-}(t, s) B_{k+}(t, s)$. Now let us rewrite the operator 
equations (16) through the kernels

$$
\begin{aligned}
& B_{k+}(t, s)+F_{k}(t, s)+\int_{-\infty}^{t} B_{k+}(t, \tau) F_{k}(\tau, s) d \tau=0, \quad s \leq t, \\
& B_{k-}(t, s)-F_{k}(t, s)-\int_{-\infty}^{t} B_{k+}(t, \tau) G_{k}(\tau, s) d \tau=0, \quad s \geq t,
\end{aligned}
$$

where $F_{k}\left(t, s\right.$, and $G_{k}(t, s)$ are the kernels of the Hilbert-Schmidt integral operators $F_{k}$ and $G_{k}=H_{k} S_{H_{k}}^{-1}-I$, respectively. These integral equations are of GLM type and uniquely solvable, which follows from factorization of the operators $S_{H_{k}}(k=1,2)$ (see $\left.[\mathbf{8}, \mathbf{1 8}]\right)$. Therefore,

$$
\begin{aligned}
& H_{k} A_{12+}-A_{22+}=\Gamma_{k+}, \\
& A_{21-}-H_{k} A_{11-}=\Gamma_{k-} H_{k},
\end{aligned}
$$

where $\Gamma_{k+}=\left[F_{k}\left(I+Q_{t} F_{k}\right)^{-1}\right]_{+}, \Gamma_{k-}=\left[G_{k}\left(I+E_{t} G_{k}\right)^{-1}\right]_{-}, k=1,2$ and $Q_{t}-$ is the projection on semi-axis when $s \leq t, E_{t}-$ is the projection on the semi-axis when $s \geq t,[K]_{+}$and $[K]_{-}$denote the lower and upper Volterra parts of the integral operator $K f(t)=$ $\int_{-\infty}^{+\infty} K(t, s) f(s) d s$, respectively, i.e., $[K]_{+} f(t)=\int_{-\infty}^{t} K(t, s) f(s) d s$ and $[K]_{-} f(t)=\int_{t}^{+\infty} K(t, s) f(s) d s$.

From (18), the transmission matrix operator $\Pi$ is uniquely determined by $S_{H_{1}}$ and $S_{H_{2}}$, under the condition $\operatorname{det}\left[H_{1}-H_{2}\right] \neq 0$. Because the matrix operator $\Pi$ uniquely determines the coefficients of system (8), then the coefficients of system (8) are uniquely determined by the scattering operators $S_{H_{1}}$ and $S_{H_{2}}$.

Remark 4.4. Theorem 4.3 is proved in [11] for the TS-structure system (see Example 3.2). In the present paper, we generalize this theorem to canonicable systems (1) with $\sigma=\operatorname{diag}\left\{\xi_{1}, \ldots, \xi_{2 m}\right\}$, where $\xi_{1} \geq \cdots \geq \xi_{m}>0>\xi_{m+1} \geq \cdots \geq \xi_{2 m}$. Theorem 4.3 is also applicable for the systems that are different from the systems in $[11,19]$. For example, the ISP on the semi-axis for the nonstrictly hyperbolic system (1) is solved where $\sigma=\operatorname{diag}\left\{\xi_{1}, \ldots, \xi_{4}\right\}, \xi_{1}=\xi_{2}>$ $0>\xi_{3}>\xi_{4}$ having the potential

$$
Q(x, t)=\left[\begin{array}{cccc}
0 & 0 & 0 & q_{14}(x, t) \\
0 & 0 & q_{23}(x, t) & q_{24}(x, t) \\
q_{31}(x, t) & q_{32}(x, t) & 0 & q_{34}(x, t) \\
q_{41}(x, t) & 0 & 0 & 0
\end{array}\right]
$$


which is not studied elsewhere. It is easy to verify that this system is canonicable, i.e., it has Volterra type integral representation in the form (4) with the kernel having structure

$$
A(x, t, s)=\left[\begin{array}{cccc}
A_{11}(x, t, s) & 0 & 0 & A_{14}(x, t, s) \\
A_{21}(x, t, s) & A_{22}(x, t, s) & A_{23}(x, t, s) & A_{24}(x, t, s) \\
A_{31}(x, t, s) & A_{32}(x, t, s) & A_{33}(x, t, s) & A_{34}(x, t, s) \\
A_{41}(x, t, s) & 0 & 0 & A_{44}(x, t, s)
\end{array}\right] .
$$

Notice that the canonicability condition (7) has the form

$$
\begin{aligned}
& q_{11}(x, t)=q_{12}(x, t)=q_{21}(x, t)=q_{22}(x, t) \\
&=q_{33}(x, t)=q_{44}(x, t)=0, \\
& q_{43}(x, t)=\frac{\xi_{3}-\xi_{4}}{\xi_{3}} \sum_{p=1}^{4} \int_{x}^{+\infty} q_{4 p}\left(\eta, t+\xi_{4}(x-\eta)\right) \\
& A_{p 3}\left(\eta, t+\xi_{k}(x-\eta), x-\frac{\xi_{4}}{\xi_{3}}(x-\eta)\right) d \eta,
\end{aligned}
$$

which is identically satisfied.

Remark 4.5. The two-velocity system ([4])

$$
\rho \frac{\partial^{2} u}{\partial t^{2}}-\frac{\partial^{2} u}{\partial x^{2}}+V(x) u=0,
$$

with $u=\operatorname{col}\left\{u_{1}, u_{2}\right\}, \rho=\operatorname{diag}\left\{\rho_{1}, \rho_{2}\right\}, 0<\rho_{1}<\rho_{2}$ and $V(x)=$ $\left[\begin{array}{ll}v_{11}(x) & v_{12}(x) \\ v_{21}(x) & v_{22}(x)\end{array}\right]$ can be easily reduced to the first order system in the form of (1):

$$
\sigma \frac{\partial \psi}{\partial t}-\frac{\partial \psi}{\partial x}=Q(x, t) \psi
$$

with $\psi=\operatorname{col}\left\{y_{1}, y_{2}, u_{1}, u_{2}\right\}, \sigma=\operatorname{diag}\left\{\xi_{1}, \xi_{2},-\xi_{2},-\xi_{1}\right\}, \xi_{1}=\rho_{2}^{1 / 2}$, $\xi_{2}=\rho_{1}^{1 / 2}$ and

$$
Q=\left[\begin{array}{cccc}
0 & 0 & q_{13} & q_{14} \\
0 & 0 & q_{23} & q_{24} \\
0 & q_{32} & 0 & 0 \\
q_{41} & 0 & 0 & 0
\end{array}\right]
$$


with

$$
\begin{gathered}
q_{13}(x, t)=\frac{v_{21}(x)}{\alpha_{1}}, \quad q_{14}=\frac{v_{22}}{\alpha_{1}}, \quad q_{23}=-\frac{v_{11}}{\alpha_{2}}, \\
q_{24}=-\frac{v_{12}}{\alpha_{2}}, \quad q_{32}=\alpha_{2}, \quad q_{41}=\alpha_{1} .
\end{gathered}
$$

Here, $\alpha_{1}$ and $\alpha_{2}$ are solutions of the equations

$$
\xi_{1} \frac{\partial \alpha_{1}}{\partial t}-\frac{\partial \alpha_{1}}{\partial x}=0 \quad \text { and } \quad \xi_{2} \frac{\partial \alpha_{2}}{\partial t}-\frac{\partial \alpha_{2}}{\partial x}=0
$$

respectively.

In general, system $(20)$ is not canonicable. When $q_{13}=0$, this system is a TS structure system; therefore, it is canonicable. Thus, Theorem 4.3 can be applied to the two-velocity system (19) on the semi-axis $x \geq 0$, since it is canonicable when $v_{21}(x)=0$.

5. Conclusion. This paper considered an interesting problem arising in one-dimensional inverse problems based on transformation operators. The method of transformation operators is well known in many diverse fields such as the area of inverse spectral problems. They also have applications in inverse scattering theory on the half line. The transformation operator for the first-order hyperbolic system at infinity has a complicated structure, and this makes it difficult to solve the ISP for that system. Because the transformation operator at infinity has a Volterra structure, the scattering operator for the canonical forms of the first order hyperbolic system on the half line have factorization in the form of (16). In this situation, it is observed that the Krein's factorization technique $([\mathbf{8}, \mathbf{1 8}])$ (it reduces ISP to the GLM type integral equation in the form of (17)) will be more applicable for the solution of ISP for the canonical forms of (8).

6. Appendix. Solvability of a system of Volterra type integral equations. In this appendix, we recall unique solvability of a system of Volterra type integral equations on the plane (see [23]). Consider $\mathbb{C}_{b}\left(\mathbb{R}^{2}\right)$ is the space of bounded continuous functions on $\mathbb{R}^{2}$. 
Lemma 6.1. (see [23, Lemma 3.1]).

(i) Let $\left(\alpha_{1}, \beta_{1}\right), \ldots,\left(\alpha_{N}, \beta_{N}\right)$ be $N$ vectors in the plane that point into the same side of a straight line, i.e., there exists $(\alpha, \beta)$ such that $\alpha \alpha_{l}+\beta \beta_{l}>0$ for $l=1, \ldots, N$.

(ii) Assume that $g_{j l}(x, y, s), 1 \leq j, l \leq N$, are continuous functions such that $\left|g_{j l}(x, y, s)\right| \leq h(\alpha x+\beta y)$ for all $(x, y) \in \mathbb{R}^{2}$, where $h \in L_{1}(\mathbb{R})$. Then the following system of integral equations

$$
\begin{gathered}
v_{j}(x, y)=f_{j}(x, y)+\int_{-\infty}^{0} \sum_{l=1}^{n}\left[g_{j l} v_{l}\right]\left(x+\alpha_{l} s, t+\beta_{l} s, s\right) d s, \\
j=1, \ldots, N,
\end{gathered}
$$

are uniquely solvable in $\mathbb{C}_{b}\left(\mathbb{R}^{2}\right)$ for any given $f_{j} \in \mathbb{C}_{b}\left(\mathbb{R}^{2}\right)$.

\section{REFERENCES}

1. I. Åberg, G. Kristensson and J.N. Wall, Propagation of transient electromagnetic waves in time-varying media-direct and inverse scattering problems, Inverse Prob. 11 (1995), 29-49.

2. M.J. Ablowitz and H. Segur, Solitons and the inverse scattering transform, SIAM, Philadelphia, 1981.

3. T. Aktosun, M. Klaus and C. van der Mee, Direct and inverse scattering for selfadjoint Hamiltonian systems on the line, Int. Equat. Oper. Theor. 38 (2000), 129-171.

4. M.I. Belishev, A. Blagovestchenskii and S.A. Ivanov, The two-velocity dynamical system: boundary control of waves and inverse problems, Wave Motion 25 (1997), 83-107.

5. M.I. Belishev and S.A. Ivanov, Characterization of data in the dynamic inverse problem for a two-velocity system Zap. Nauch. St.-Peterburg. Otdel. Mat. Inst. Stek. (POMI) 259 (1999); J. Math. Sci. 109 (2002), 1814-1834 (in English).

6. F. Demontis and C. van der Mee, Marchenko equations and norming constants of the matrix Zakharov-Shabat system, Op. Matr. 2 (2008), 79-113.

7. M.G. Gasymov, The inverse scattering problem for a system of Dirac equations of order 2n, Moscow Math. Soc. 19 (1968), 41-119.

8. I. Gohberg and M. Krein, Theory and applications of Volterra operators in Hilbert space, American Mathematical Society, Providence, RI, 1970.

9. N.Sh. Iskenderov and M. I. Ismailov, Inverse scattering problem for nonstationary Dirac-type systems on the half-plane, J. Diff. Equat. 246 (2009), 277-290.

10. On the inverse scattering transform of a nonlinear evolution equation with $2+1$ dimensions related to nonstrict hyperbolic systems, Nonlinearity $\mathbf{2 5}$ (2012), 1967-1979. 
11. M.I. Ismailov, Inverse scattering problem for hyperbolic system on a semiaxis in the case of equal number of incident and scattered waves, Inverse Prob. 22 (2006), 955-974.

12. , Inverse scattering problem for nonstationary Dirac-type systems on the plane, J. Math. Anal. Appl. 365 (2010), 498-509.

13. M. Lesch and M.M. Malamud, The inverse spectral problem for first order systems on the half line, Op. Th. Adv. Appl. 117, Birkhäuser, Basel, 2000.

14. B.M. Levitan, Inverse Sturm-Liouville problems, VNU Science Press, Utrecht, 1987.

15. S.V. Manakov, On the theory of two-dimensional stationary self focussing of electromagnetic waves, Sov. Phys. JETP 38 (1974), 248-253.

16. V.A. Marchenko, Sturm-Liouville operators and applications, AMS Chelsea Publishing, Philadelphia, 2011.

17. A. Morassi, G. Nakamura and M. Sini, An inverse dynamical problem for connected beams, Eur. J. Appl. Math. 16 (2005), 83-109.

18. L.P. Nizhnik, Inverse scattering problems for hyperbolic equations, Nauk. Dumk., Kiev, 1991 (in Russian).

19. L.P. Nizhnik and N.Sh. Iskenderov, An inverse nonstationary scattering problem for a hyperbolic system of three first-order equations on the half-axis, Ukr. Mat. Zh. 42 (1990), 931-938.

20. Rakesh and Paul Sacks, Stability for an inverse problem for a two-speed hyperbolic PDE in one space dimension, Inv. Prob. 26 (2010), 025005 (20 pages).

21. A.L. Sakhnovich, Dirac type system on the axis: Explicit formulae for matrix potentials with singularities and soliton-positon interactions, Inv. Prob. 19 (2003), 845-854.

22. L.A. Sakhnovich, Spectral theory of canonical differential systems. Method of operator identities, Birkhäuser, Basel, 1999.

23. L.Y. Sung and A.S. Fokas, Inverse problem for $N \times N$ hyperbolic systems on the plane and N-wave interactions, Comm. Pure Appl. Math. 44 (1991), 535-571.

24. A.E. Yagle, Inverse scattering for time-varying one-dimensional layered media: Algorithms and applications, Inv. Prob. 13 (1997), 867-881.

25. V.A. Yurko, An inverse spectral problem for singular non-self-adjoint differential systems, Mat. Sb. 195 (2004), 1823-1854 (in Russian); Sb. Math. 195 (2004), 123-156 (in English).

Department of Mathematics, Gebze Institute of Technology, Gebze-Kocaeli 41400, Turkey and Institute of Mathematics and Mechanics, National Academy of Sciences of Azerbaijan, Baku AZ1141, Azerbaijan

Email address: mismailov@gyte.edu.tr

Department of Mathematics, Gebze Institute of Technology, Gebze-Kocaeli 41400, TURKEY

Email address: itekin@gyte.edu.tr 